IFP-432-UNC

hep-th/9205094

\title{
Running Gauge Couplings and Thresholds in the Type II Superstring
}

\author{
L. Dolan and James T. Liu \\ Institute of Field Physics \\ Department of Physics and Astronomy \\ University of North Carolina \\ Chapel Hill, NC 27599-3255, USA
}

\begin{abstract}
A distinctive feature of string unification is the possibility of unification by a nonsimply-laced group. This occurs most naturally in four dimensional type II string models where the gauge symmetry is realized by Kac-Moody algebras at different levels. We investigate the running coupling constants and the one-loop thresholds for such general models. As a specific case, we examine a $\mathrm{SU}(3) \times \mathrm{U}(1) \times \mathrm{U}(1)$ model and find that the threshold corrections lead to a small $6 \%$ increase in the unification scale.
\end{abstract}

May 1992 


\section{Introduction}

There has been much excitement over the recent precision electroweak measurements at LEP and their implication for grand unification. In particular, this data shows that with a given normalization, corresponding to grand unification of the standard model via a simply laced group, the running coupling constants become equal at a GUT scale of the order $10^{16} \mathrm{GeV}[\mathbf{1}[3]$.

In this paper, we are interested in the alternative possibility that the low-energy physics is unified by a string theory. At first glance, string theory, which is perhaps the only known consistent theory of gravity, does not predict any running of the couplings since it is a finite theory. However, when we only look at the massless string modes, then they can be described by an effective field theory. It is in this context that we talk about running coupling constants in string theory 四, 5].

Since the string scale is set by the Planck mass, it appears that string unification would occur at the Planck scale. For the heterotic string, this seems to contradict the possible evidence of unification at $10^{16} \mathrm{GeV}$. However, explicit calculations of threshold effects at the string scale show that these contributions may be large enough to lower the unification scale to a more phenomenologically acceptable regime. This is understood because, while a single massive state alone may not give rise to a large threshold contribution, a string theory has an infinite tower of massive levels, all of which will contribute.

Although previous investigations of string unification have focused on heterotic models[6 [13], we wish to carry out the analysis in the context of four dimensional type II superstring models. Our reason for doing so is that one of the goals of string theory is to find a theory with no or few parameters. While the heterotic string has a very rich structure, it has perhaps too much freedom and gives rise to a plethora of possible vacuum states. The type II models are a lot more economical (perhaps too much so[14]) and thus furnish a simpler and more constrained "testing ground" for string theory. Even if type II models turn out to be unrealistic, we feel it is an ideal testbed for working out techniques of low-energy string phenomenology because of its relative simplicity.

In order to talk about running coupling constants, we describe the massless string states by an effective field theory where the massive states have been integrated out. We are then concerned with matching this low-energy theory with the fundamental string theory. The tree-level relation between the low-energy coupling constants [15] is $g_{i}^{2}=2 g_{\mathrm{str}}^{2} / x_{i}$ where the factor of 2 arises because we choose a field theory normalization for the length squared 
of the longest roots equal to 1 and where $x_{i}$ is the level of the Kac-Moody algebra (KMA). Using $\alpha_{i}=g_{i}^{2} / 4 \pi$, the one-loop formula for the running couplings is

$$
\frac{1}{\alpha_{i}(\mu)}=\frac{x_{i} / 2}{\alpha_{\mathrm{GUT}}}-\frac{b_{i}}{2 \pi} \log \mu / M_{\mathrm{str}}+\frac{\Delta_{i}}{4 \pi}
$$

where $b_{i}$ are the $\beta$-function coefficients calculated in the effective field theory and $\Delta_{i}$ are threshold corrections arising from the matching conditions. $M_{\mathrm{str}}$ is an effective string unification scale and $\alpha_{\mathrm{GUT}}$ is an effective coupling which will be related to the string coupling constant below.

In the following, we calculate the $\Delta_{i}$ for general type II models which naturally include KMA representations with $x_{i}>1$. We use the background field method[16] to calculate the one-loop effects, and our calculation parallels that of Kaplunovsky[6]. Finally, we look at a specific example and calculate the thresholds numerically for an $N=2 \mathrm{SU}(3) \times \mathrm{U}(1) \times \mathrm{U}(1)$ model. We find that the correction only amounts to a small shift of the unification mass scale and conclude that thresholds may have a smaller effect in type II theories than in heterotic models.

\section{Background Field Calculation}

We study the one-loop renormalization of the gauge coupling constants through the background field method. This allows us to quantize the gauge field by treating it as a classical background in which the strings propagate. We note that this approach is different from that of the conventional "strings in background fields" work [17]. The standard work focuses on studying the consistent propagation of strings in curved space and background gauge fields. This is achieved when the string sigma-model $\beta$-functions vanish and the model is conformally invariant. For that work, it is sufficient to look at the string tree level. On the other hand, we are concerned with the space-time gauge coupling constant $\beta$-function which we calculate at the string one-loop level.

Our starting point is the string sigma-model describing the propagation of strings in a background gauge field. The string effective action in the presence of an $A_{\mu}$ background can be represented as $\Gamma_{\sigma-\operatorname{model}}\left[X^{\mu}, \psi^{\mu} ; A_{\mu}\right]$ where $X^{\mu}$ and $\psi^{\mu}$ are the string fields. The background field method [16] tells us that the effective action for the gauge field is given by $\Gamma\left[A_{\mu}\right]=\Gamma_{\sigma-\text { model }}\left[X^{\mu}=0, \psi^{\mu}=0 ; A_{\mu}\right]$ where $\Gamma\left[A_{\mu}\right]=\int d^{4} x \mathcal{L}\left(A_{\mu}\right)$. In other words, the gauge field effective action is given by the partition function of the string in the presence of that gauge field. 
At tree level, the effective lagrangian $\mathcal{L}\left(A_{\mu}\right)$ is simply the classical Lagrangian

$$
\mathcal{L}_{\text {tree }}\left(A_{\mu}\right)=\sum_{i}-\frac{1}{4 g_{i}^{2}} F_{\mu \nu}^{a} F^{\mu \nu a}
$$

where $i$ labels the subgroup. We are interested in the one-string-loop correction to this tree level result. For simplicity, we examine each subgroup independently. For subgroup $i$, we turn on a constant background field-strength, $A_{\mu}^{i}(X)=-\frac{1}{2} F_{\mu \nu} X^{\nu}$, where $F_{\mu \nu}$ is a constant $\left(A_{\mu}^{j}(X)=0\right.$ for $\left.j \neq i\right)$. If the subgroup is nonabelian, it is sufficient to shift only one of the gauge fields in the subgroup because of gauge invariance. We set all other components in that subgroup to zero so $F_{\mu \nu}$ above carries no internal gauge index and is abelian. Although this background gauge field will induce a background gravitational field via Einstein's equations, since we are only interested in the gauge group dependent thresholds, it is sufficient to calculate the two-point background gauge field amplitude[6]. The coefficient of $-\frac{1}{4} F_{\mu \nu}^{2}$ in this amplitude is then the one-loop correction to the tree-level gauge coupling $\frac{1}{g_{i}^{2}}$.

The four dimensional type II string models [18 21] are constructed out of 20 leftmoving (2 space-time and 18 internal) and 20 right-moving light-cone gauge free fermions. A particular model is described by a set of boundary conditions, $\Omega$, for the 40 fermions. The partition function is then given as a sum over the sectors (labeled by $\alpha$ and $\beta$ ) generated by the set of $\rho_{\alpha} \equiv\left(\rho_{\alpha} ; \bar{\rho}_{\alpha}\right) \in \Omega$

$$
\begin{aligned}
Z & =\frac{1}{2^{K+1}} \sum_{\alpha, \beta} c(\alpha, \beta) \operatorname{Tr}_{\alpha}\left[q^{L_{0}^{\prime}-\alpha_{0}} \bar{q}^{\bar{L}_{0}^{\prime}-\bar{\alpha}_{0}}(-1)^{N_{\beta}}\right] \\
& =\frac{1}{2^{K+1}} \sum_{\alpha, \beta} c(\alpha, \beta)|\eta(q)|^{-24} \prod_{i=1}^{20}\left(\vartheta\left[\begin{array}{c}
\rho_{\alpha}^{i} \\
\rho_{\beta}^{i}
\end{array}\right](q)\right)^{1 / 2} \prod_{i=1}^{20}\left(\vartheta\left[\begin{array}{c}
\bar{\rho}_{\alpha}^{i} \\
\bar{\rho}_{\beta}^{i}
\end{array}\right](\bar{q})\right)^{1 / 2}
\end{aligned}
$$

where $\Omega$ is generated by $K+1$ independent vectors. The $c(\alpha, \beta)=\delta_{\alpha} \epsilon(\alpha, \beta)$ are phases for the $(-1)^{N_{\beta}}$ projections as described in [19]. In the above, the prime denotes the omission of the bosonic zero mode, $p$.

We work in the operator formalism in order to fix the various normalizations. The oneloop two-point amplitude contribution to the effective lagrangian for the $A_{\mu}^{i}$ background can be written in this operator language as

$$
\mathcal{L}^{\prime}\left(A_{\mu}^{i}\right)=\frac{1}{2^{K+1}} \sum_{\alpha, \beta} c(\alpha, \beta) \int \frac{d^{4} p}{(2 \pi)^{4}} \operatorname{Tr}_{\alpha}\left[\Delta V^{i}(1,1) \Delta V^{i}(1,1)(-1)^{N_{\beta}}\right]
$$


where the sum over sectors corresponds to a generalized GSO projection 19. The closed string propagator is

$$
\Delta=\frac{\alpha^{\prime}}{2 \pi} \int_{|z| \leq 1} \frac{d z d \bar{z}}{|z|^{2}} z^{L_{0}-\alpha_{0}} \bar{z}^{\bar{L}_{0}-\bar{\alpha}_{0}} .
$$

Although this is written in the (NS,NS) sector where $\alpha_{0}=\bar{\alpha}_{0}=1 / 2$, other spin structures are treated similarly. In general, $\alpha_{0}=-1 / 12-b / 48+d / 24$ where $(b, d)$ is the total number of NS and R left moving fermions respectively in a given sector, and $\bar{\alpha}_{0}$ is similarly defined for right moving fermions.

The $V^{i}(1,1)$ are conformal weight $(1,1)$ background field vertices. They are created from the ordinary type II vertex (in covariant gauge) for emission of a gauge boson with state $\tilde{b}_{-1 / 2}^{a} \epsilon \cdot b_{-1 / 2}|k\rangle$

$$
\begin{aligned}
V^{a}(k, \epsilon, z, \bar{z})= & {\left[\frac{1}{2} \sqrt{2 \alpha^{\prime}} k \cdot \tilde{\psi}(z) \tilde{\psi}^{a}(z)-\frac{i}{2} f_{a b c} \tilde{\psi}^{b}(z) \tilde{\psi}^{c}(z)\right] } \\
& \epsilon \cdot\left[i \bar{z} \bar{\partial} X_{\mathrm{R}}(\bar{z})-\frac{1}{2} \sqrt{2 \alpha^{\prime}} \psi(\bar{z}) k \cdot \psi(\bar{z})\right] e^{i \sqrt{2 \alpha^{\prime}} k \cdot X(z, \bar{z})}
\end{aligned}
$$

by the substitution $\epsilon_{\mu} e^{i \sqrt{2 \alpha^{\prime}} k \cdot X} \rightarrow A_{\mu}(X)$ provided $A_{\mu}$ solves the equations of motion, $\partial_{\mu} F^{\mu \nu}=0$. For a constant $F_{\mu \nu}$ corresponding to a given component of subgroup $i$, the resulting background field vertex is

$$
\begin{aligned}
V^{i}\left[F_{\mu \nu}\right](z, \bar{z})=\frac{i}{4} F_{\mu \nu} & \left\{J^{a}(z)\left[2 X^{\mu}(z, \bar{z}) \bar{z} \bar{\partial} X_{\mathrm{R}}^{\nu}(\bar{z})-\psi^{\mu}(\bar{z}) \psi^{\nu}(\bar{z})\right]\right. \\
& \left.-i\left[\tilde{\psi}^{\mu}(z) \tilde{\psi}^{a}(z)\right]\left[\bar{z} \bar{\partial} X_{\mathrm{R}}^{\nu}(\bar{z})\right]\right\} \delta^{a i}
\end{aligned}
$$

Here,

$$
\begin{aligned}
X^{\mu}(z, \bar{z}) & =\frac{x^{\mu}}{\sqrt{2 \alpha^{\prime}}}+\frac{\sqrt{2 \alpha^{\prime}} p^{\mu}}{4 i}(\ln z+\ln \bar{z})+\frac{i}{2} \sum_{n \neq 0} \frac{1}{n} \tilde{\alpha}_{n}^{\mu} z^{-n}+\frac{i}{2} \sum_{n \neq 0} \frac{1}{n} \alpha_{n}^{\mu} \bar{z}^{-n} \\
& =\frac{1}{2}\left(X_{\mathrm{L}}^{\mu}(z)+X_{\mathrm{R}}^{\mu}(\bar{z})\right)
\end{aligned}
$$

and

$$
J^{a}(z)=-\frac{i}{2} f_{a b c} \tilde{\psi}^{b}(z) \tilde{\psi}^{c}(z)
$$

is the quark model current satisfying a KMA

$$
J^{a}(z) J^{b}(w)=\frac{k_{i} \delta^{a b}}{(z-w)^{2}}+\frac{i f_{a b c}}{z-w} J^{c}(w)+\text { regular terms }
$$

with $k_{i}=C_{\psi} / 2$. Since the $f_{a b c}$ are normalized by $f_{a b c} f_{a b e}=C_{\psi} \delta_{c e}=2 \delta_{c e}$, all $k_{i}=1$. This value of $k_{i}$ is dependent on the root normalization whereas the level of the KMA, given by $x_{i}=2 k_{i} \tilde{h}_{i} / C_{\psi}$ for $\tilde{h}_{i}$ the dual Coxeter number of a given subgroup defined by $\tilde{h}=C_{\psi} / \psi^{2}$ 
with $\psi^{2}$ being the length squared of the longest root in the subgroup $(\tilde{h}=N$ for $\mathrm{SU}(N))$, is independent of any normalization. This point is important when we wish to compare the string theory results where $C_{\psi}=2$, corresponding to $\psi_{i}^{2}=2 / \tilde{h}_{i}$, with the conventional field theory results which are given in a normalization with all roots $\psi_{i}^{2}=1$. Written in this manner, the first line in (2.6) is identical to the heterotic background vertex [6] except that $J^{a}$, given by (2.8), is no longer a level 1 representation. The additional term in (2.6) is due to the gauge group being generated by a super KMA in the present case of the type II string. The $\delta^{a i}$ in (2.6) denotes that we only turn on a single component of the background gauge field in each subgroup. Because of gauge invariance, it is unimportant which specific component we choose.

Using standard operator methods, we rewrite (2.3) as

$$
\begin{aligned}
\mathcal{L}^{\prime}\left(A_{\mu}^{i}\right)=\frac{1}{2^{K+1}} \sum_{\alpha, \beta} c(\alpha, \beta)\left(2 \alpha^{\prime} \pi\right)^{2} \int & \frac{d^{4} p}{(2 \pi)^{4}} \int_{\Gamma} d^{2} \tau \int_{0 \leq \operatorname{Im} \nu \leq \operatorname{Im} \tau} d^{2} \nu \\
& \operatorname{Tr}_{\alpha}\left[V^{i}(z, \bar{z}) V^{i}(1,1) q^{L_{0}-\alpha_{0}} \bar{q}^{\bar{L}_{0}-\bar{\alpha}_{0}}(-1)^{N_{\beta}}\right]
\end{aligned}
$$

where $z=e^{2 \pi i \nu}$ and $q=e^{2 \pi i \tau}$ and we have restricted the modular integral over the fundamental region $\Gamma$. The operator trace gives the two-point correlator $\langle V V\rangle$ on the torus

$$
\begin{aligned}
\left\langle V^{i}(z, \bar{z}) V^{i}(w, \bar{w})\right\rangle_{\alpha, \beta} \equiv \frac{\operatorname{Tr}_{\alpha}\left[V^{i}(z, \bar{z}) V^{i}(w, \bar{w}) q^{L_{0}-\alpha_{0}} \bar{q}^{L_{0}-\bar{\alpha}_{0}}(-1)^{N_{\beta}}\right]}{\operatorname{Tr}_{\alpha}\left[q^{L_{0}-\alpha_{0}} \bar{q}^{\left.\bar{L}_{0}-\bar{\alpha}_{0}(-1)^{N_{\beta}}\right]}\right.} \\
=-\frac{1}{16} F_{\mu \nu} F_{\rho \sigma}\left\{\left\langle J^{i}(z) J^{i}(w)\right\rangle\right. \\
\times\left[\left\langle 2 X^{\mu}(z, \bar{z}) \bar{z} \bar{\partial} X_{\mathrm{R}}^{\nu}(\bar{z}) 2 X^{\rho}(w, \bar{w}) \bar{w} \bar{\partial} X_{\mathrm{R}}^{\sigma}(\bar{w})\right\rangle\right. \\
\left.+\left\langle\psi^{\mu}(\bar{z}) \psi^{\nu}(\bar{z}) \psi^{\rho}(\bar{w}) \psi^{\sigma}(\bar{w})\right\rangle\right] \\
\left.-\left\langle\tilde{\psi}^{\mu}(z) \tilde{\psi}^{i}(z) \tilde{\psi}^{\rho}(w) \tilde{\psi}^{i}(w)\right\rangle\left\langle\bar{z} \bar{\partial} X_{\mathrm{R}}^{\nu}(\bar{z}) \bar{w} \bar{\partial} X_{\mathrm{R}}^{\sigma}(\bar{w})\right\rangle\right\} .
\end{aligned}
$$

After dropping total derivatives, we find that the last term above, which comes from the additional term in (2.6), does not contribute to $\mathcal{L}^{\prime}\left(A_{\mu}^{i}\right)$. Performing the $p$ integration then gives

$$
\begin{aligned}
\mathcal{L}^{\prime}\left(A_{\mu}^{i}\right)=\frac{1}{4} F_{\mu \nu}^{2} & \frac{1}{16 \pi^{2}} \frac{1}{2^{K+1}} \sum_{\alpha, \beta} c(\alpha, \beta) \int_{\Gamma} \frac{d^{2} \tau}{\tau_{2}} \int \frac{d^{2} \nu}{\tau_{2}}\left\langle J^{i}(z) J^{i}(1)\right\rangle_{\alpha, \beta} \\
& \times 2\left[\langle\psi(\bar{z}) \psi(1)\rangle_{\alpha, \beta}^{2}-\left\langle X_{\mathrm{R}}(\bar{z}) \bar{z} \bar{\partial} X_{\mathrm{R}}(1)\right\rangle^{2}\right] \operatorname{Tr}_{\alpha}\left[q^{L_{0}^{\prime}-\alpha_{0}} \bar{q}^{\bar{L}_{0}^{\prime}-\bar{\alpha}_{0}}(-1)^{N_{\beta}}\right]
\end{aligned}
$$


which is similar to the expression for the heterotic case where we have corrected several numerical factors in [6].

Using (2.9), we find that

$$
\left\langle J^{i}(z) J^{i}(1)\right\rangle=-k_{i}\left(z \frac{\partial}{\partial z}\right)^{2} \log \vartheta_{1}(z, q)+\left\langle J_{0}^{i} J_{0}^{i}\right\rangle .
$$

As pointed out in [6], the first term yields a gauge group independent (apart from the $k_{i}$ ) term which can be combined with the induced gravitational background term. We thus drop this piece and concentrate on the group dependent charges $\left\langle J_{0}^{i} J_{0}^{i}\right\rangle$. For the space-time correlators, both $\langle\psi \psi\rangle^{2}$ and $\left\langle X_{\mathrm{R}} \bar{z} \bar{\partial} X_{\mathrm{R}}\right\rangle^{2}$ have double poles in the $\nu$ plane and are related to the Weierstrass $\mathcal{P}$ function. As a result, the difference is finite, so the $\nu$ integral can be performed to get

$$
\begin{aligned}
\mathcal{L}^{\prime}\left(A_{\mu}^{i}\right)=\frac{1}{4} F_{\mu \nu}^{2} \frac{1}{16 \pi^{2}} & \frac{1}{2^{K+1}} \sum_{\alpha, \beta} c(\alpha, \beta) \int_{\Gamma} \frac{d^{2} \tau}{\tau_{2}} 2\left\langle J_{0}^{i} J_{0}^{i}\right\rangle_{\alpha, \beta} \\
& \times 2 \bar{q} \frac{d}{d \bar{q}} \log \left(\vartheta\left[\begin{array}{c}
\bar{\rho}_{\alpha}^{1} \\
\bar{\rho}_{\beta}^{1}
\end{array}\right](\bar{q}) / \eta(\bar{q})\right) \operatorname{Tr}_{\alpha}\left[q^{L_{0}^{\prime}-\alpha_{0}} \bar{q}^{\prime} \bar{L}_{0}^{\prime}-\bar{\alpha}_{0}(-1)^{N_{\beta}}\right] .
\end{aligned}
$$

Since all space-time fermions are moded identically, we have arbitrarily picked the boundary condition of the first fermion.

At this stage, the expression is equivalent to that for the heterotic string. However, for the type II string, we can now use the explicit realization (2.8) of the $J^{a}(z)$ in terms of free fermions in order to evaluate $\left\langle J_{0}^{i} J_{0}^{i}\right\rangle$ explicitly. For a symmetric subgroup, both fermions generating $J_{0}^{a}$ are moded identically, and the result is

$$
\left\langle J_{0}^{i} J_{0}^{i}\right\rangle=\frac{1}{2} f^{i}{ }_{c d} f^{i}{ }_{c d} 2 q \frac{d}{d q} \log \vartheta\left[\begin{array}{c}
\rho_{\alpha}^{c} \\
\rho_{\beta}^{c}
\end{array}\right](q)
$$

(no sum on $i$ ). The spin structure is that of fermion $c$ (or equivalently $d$ ). Using the form of the partition function, (2.2), we finally arrive at the expression

$$
\mathcal{L}^{\prime}\left(A_{\mu}^{i}\right)=-\frac{1}{4} F_{\mu \nu}^{2} \frac{1}{16 \pi^{2}} \int_{\Gamma} \frac{d^{2} \tau}{\tau_{2}} 2 B_{i}(q, \bar{q})
$$

where

$$
\begin{aligned}
B_{i}(q, \bar{q})=- & \frac{1}{2^{K+1}} \sum_{\alpha, \beta} c(\alpha, \beta)|\eta(q)|^{-23} \vartheta\left[\begin{array}{c}
\rho_{\alpha}^{1} \\
\rho_{\beta}^{1}
\end{array}\right](q) 2 \bar{q} \frac{d}{d \bar{q}}\left(\vartheta\left[\begin{array}{c}
\bar{\rho}_{\alpha}^{1} \\
\bar{\rho}_{\beta}^{1}
\end{array}\right](\bar{q}) / \eta(\bar{q})\right) \\
& \times \prod_{j=3}^{20}\left(\vartheta\left[\begin{array}{c}
\rho_{\alpha}^{j} \\
\rho_{\beta}^{j}
\end{array}\right](q)\right)^{1 / 2} \prod_{j=3}^{20}\left(\vartheta\left[\begin{array}{c}
\bar{\rho}_{\alpha}^{j} \\
\bar{\rho}_{\beta}^{j}
\end{array}\right](\bar{q})\right)^{1 / 2} \frac{1}{2} f^{i}{ }_{c d} f^{i}{ }_{c d} 2 q \frac{d}{d q} \log \vartheta\left[\begin{array}{c}
\rho_{\alpha}^{c} \\
\rho_{\beta}^{c}
\end{array}\right](q) .
\end{aligned}
$$


Recall that the one-loop renormalization to $\frac{1}{g_{i}^{2}}$ is the coefficient of $-\frac{1}{4} F_{\mu \nu}^{2}$. We now want to match this string theory result to that of the low-energy effective field theory of the massless particles in four dimensions [6,22]. In order to match the normalizations for nonabelian groups, we have to convert from the type II string normalization of $C_{\psi}=2$ to the field theory convention of $\psi_{\mathrm{FT}}^{2}=1$. This gives an overall factor of $\psi_{\mathrm{FT}}^{2} / \psi_{\mathrm{str}}^{2}=\tilde{h}_{i} / 2=$ $x_{i} / 2$ for subgroup $i$. For abelian groups, the level is undefined. In this case, we can choose $x_{\mathrm{U}(1)}=2$ which corresponds to using the same normalization for the $\mathrm{U}(1)$ charges in field theory and string theory. Equating the bare couplings then gives

$$
\frac{16 \pi^{2}}{g_{i}^{2}}+b_{i} \int_{0}^{\infty} \frac{d t}{t} C_{\Lambda}(t)=\frac{x_{i}}{2}\left[\frac{16 \pi^{2}}{g_{\mathrm{str}}^{2}}+\int_{\Gamma} \frac{d^{2} \tau}{\tau_{2}} 2 B_{i}(q, \bar{q})+Y\right]
$$

where $C_{\Lambda}(t)$ is a field theory ultraviolet cutoff. $Y$ is the gauge group independent terms that we have not evaluated. The effect of $Y$ is to shift the string coupling constant and can be absorbed by a redefinition of the unified coupling constant 1

$$
\frac{1}{\alpha_{\mathrm{GUT}}}=\frac{4 \pi}{g_{\mathrm{str}}^{2}}+\frac{Y}{4 \pi} \text {. }
$$

The $b_{i}$ are the field theory $\beta$-functions and agree with the string calculation

$$
b_{i}=\lim _{q \rightarrow 0} x_{i} B_{i}(q, \bar{q})=-\frac{11}{3} \operatorname{Tr}_{\mathrm{V}}\left(Q_{i}^{2}\right)+\frac{2}{3} \operatorname{Tr}_{\mathrm{F}}\left(Q_{i}^{2}\right)+\frac{1}{6} \operatorname{Tr}_{\mathrm{S}}\left(Q_{i}^{2}\right)
$$

(in field theory normalization). Here, the traces are over two-component fermions and real scalers.

Using $\alpha_{i}=g_{i}^{2} / 4 \pi$ and following [6] in converting (2.18) into an expression for the $\overline{\mathrm{DR}}$ couplings, we finally arrive at the matching formula, (1.1), for the running couplings

$$
\frac{1}{\alpha_{i}(\mu)}=\frac{x_{i} / 2}{\alpha_{\mathrm{GUT}}}-\frac{b_{i}}{2 \pi} \log \mu / M_{\mathrm{str}}+\frac{\Delta_{i}}{4 \pi} .
$$

The thresholds are

$$
\Delta_{i}=\int_{\Gamma} \frac{d^{2} \tau}{\tau_{2}}\left[x_{i} B_{i}(q, \bar{q})-b_{i}\right]
$$

1 Strictly speaking, modular anomalies arise in the above expressions because the first term of (2.13) has double poles on the world-sheet and must be regulated[7]. However, the differences between the gauge couplings, which are our main interest, are indeed modular invariant. As a result, $\alpha_{\mathrm{GUT}}$ itself should only be viewed as a formal expression. 
and the string unification scale is

$$
M_{\mathrm{str}}^{2}=\frac{2 e^{(1-\gamma)}}{\sqrt{27} \pi \alpha^{\prime}}
$$

where $\gamma$ is the Euler constant. Because of the string relation, $\kappa=\frac{1}{2} g_{\text {st }} \sqrt{2 \alpha^{\prime}}$, the scale $M_{\text {str }}$ can be related in the $\overline{\mathrm{DR}}$ scheme to the string coupling constant with the result $[6,8]$

$$
M_{\mathrm{str}}=0.7 \times g_{\mathrm{str}} \times 10^{18} \mathrm{GeV}
$$

Such a constraint on the unification scale is an additional feature of string unification that is not found in conventional grand unification.

\section{An $N=2$ model}

For an explicit example of how this formalism works, we now turn to specific type II models. For the original $N=4$ models with dimension 18 gauge groups $\mathrm{SU}(2)^{6}, \mathrm{SU}(4) \times$ $\mathrm{SU}(2)$ or $\mathrm{SU}(3) \times \mathrm{SO}(5)$, eight right-moving fermions are moded identically, and the $\beta$ functions and thresholds both vanish by $N=4$ supersymmetry as in [6]. This result is easily interpreted by realizing that $N=4$ gives a finite supersymmetric gauge field theory.

While as of yet there are no realistic type II string models, the $N=2 \mathrm{SU}(3) \times \mathrm{U}(1) \times$ $\mathrm{U}(1)$ model of 19] has some interesting features. This model is given by $K=2$ and is generated by the basis 19

$$
\begin{aligned}
& \rho_{b_{0}}=\left((1)^{12},(1)^{8} ;(1)^{4},(1)^{8},(1)^{4},(1)^{4}\right) \\
& \rho_{b_{1}}=\left((1)^{12},(0)^{8} ;(1)^{4},(1)^{8},(0)^{4},(0)^{4}\right) \\
& \rho_{b_{2}}=\left((1)^{12},(1)^{8} ;(0)^{4},(1)^{8},(1)^{4},(0)^{4}\right) .
\end{aligned}
$$

The massless states come from the sectors with four or less Ramond fermions on each side. These are given by the boundary conditions $\emptyset, b_{0} b_{1}, b_{0} b_{2}$ and $b_{1} b_{2}$. The states from the bosonic and the fermionic sectors combine to form an $N=2$ supergravity multiplet with helicities $( \pm 2,2( \pm 3 / 2), \pm 1)$, an $N=2$ super-Yang-Mills multiplet with helicities $( \pm 1,2( \pm 1 / 2), 2(0))$ in a singlet and adjoint representation representation of the gauge group, and $N=2$ matter with helicities $(2( \pm 1 / 2), 4(0))$ in the $\mathrm{SU}(3) \times \mathrm{U}(1) \times \mathrm{U}(1)$ representations

$$
\begin{aligned}
& {[\mathbf{1}, 0, \pm 1] \oplus[\mathbf{3},-1 / \sqrt{3}, 0] \oplus[\overline{\mathbf{3}}, 1 / \sqrt{3}, 0]} \\
& \quad \oplus\left[\mathbf{1}, \sqrt{3} / 2, \pm \frac{1}{2}\right] \oplus\left[\mathbf{1},-\sqrt{3} / 2, \pm \frac{1}{2}\right] \oplus\left[\mathbf{3}, 1 /(2 \sqrt{3}), \pm \frac{1}{2}\right] \oplus\left[\overline{\mathbf{3}},-1 /(2 \sqrt{3}), \pm \frac{1}{2}\right] .
\end{aligned}
$$


This model arises from the $N=4 \mathrm{SU}(4) \times \mathrm{SU}(2)$ model by considering the symmetric subgroups $\mathrm{SU}(3) \times \mathrm{U}(1)$ and $\mathrm{U}(1)$ of $\mathrm{SU}(4)$ and $\mathrm{SU}(2)$ respectively. As a result, the $\mathrm{SU}(3)$ is in a level 4 representation of the KMA so $x_{i}=(4,2,2)$ where we have used the natural normalization that arises from the string theory for the $U(1)$ factors. The field theory $\beta$-functions, (2.20), are easily calculated from the massless spectrum. The result is $b_{i}=(0,12,12)$. Since the $\mathrm{SU}(3) \beta$-function vanishes at one-loop, it actually vanishes at all orders because this is a sufficient condition for finiteness of the $N=2$ super-Yang-Mills coupled to $N=2$ matter theory [23].

Using the explicit form of the $\mathrm{SU}(4) \times \mathrm{SU}(2)$ structure functions, we see that the fermions generating the gauge group are either both moded like the first twelve or the last eight left-moving world-sheet fermions. As a result, we find that $B_{\mathrm{SU}(3)}(q, \bar{q})=$ $3 / 4 B_{A}(q, \bar{q})+1 / 4 B_{\alpha}(q, \bar{q})$ and $B_{\mathrm{U}(1)}(q, \bar{q})=B_{\alpha}(q, \bar{q})$ where $A$ refers to the first, and $\alpha$ refers to the last set of boundary conditions. Both $\mathrm{U}(1) \beta$-functions and thresholds are identical at the string level.

In order to calculate $B_{A}(q, \bar{q})$ and $B_{\alpha}(q, \bar{q})$, we expand the $\vartheta$-functions in (2.17) in terms of $q$ and perform a numerical integration over the fundamental region. Since $\tau_{2} \geq \sqrt{3} / 2$ in the fundamental region, $|q| \leq e^{-\sqrt{3} \pi} \approx 0.0043$, so the series expansions converge rapidly. While only sectors with massless particles contribute to the $\beta$-functions, all sectors contribute to the massive string thresholds. We numerically evaluate each sector independently taking care to combine differences of $\vartheta$-functions analytically to minimize numerical errors in the limit $\tau_{2} \rightarrow \infty$. The actual double integration is performed by open Romberg integrations in $\tau_{1}$ and $\tau_{2}$. Because $B_{i}\left(-\tau_{1}, \tau_{2}\right)=B_{i}\left(\tau_{1}, \tau_{2}\right)^{*}$ where $*$ denotes the complex conjugate, it is sufficient to integrate twice the real part of $B_{i}$ over half of the fundamental region. The thresholds are, of course, real. The result of this numerical integration is the thresholds $\Delta_{i}=(-2.35,0.144,0.144)$.

Since there are only two independent running couplings, we define the effective unification scale, $M_{\mathrm{U}}$, to be where the string theory normalized couplings meet, i.e. $1 / \alpha_{i}\left(M_{\mathrm{U}}\right)=$ $\left(x_{i} / 2\right) / \alpha_{\mathrm{GUT}}$. The difference, $\Delta_{\mathrm{SU}(3)}-\Delta_{\mathrm{U}(1)}=-1.32$ (in string theory normalization), then corresponds to an increase of the effective unification scale over $M_{\text {str }}$ of

$$
M_{\mathrm{U}} / M_{\mathrm{str}}=\exp \left(\frac{1}{2} \frac{\Delta_{\mathrm{SU}(3)}-\Delta_{\mathrm{U}(1)}}{b_{\mathrm{SU}(3)}-b_{\mathrm{U}(1)}}\right)=\exp \left(\frac{1.32}{24}\right)=1.06 .
$$

This $6 \%$ increase is rather small compared to estimates made for some heterotic models [9]. It is difficult to interpret this result physically since this is not a particularly realistic 
model. However, this leads us to believe that thresholds may play a smaller role in type II string unification than it does in the heterotic case.

\section{Conclusion}

In general orbifold models of the heterotic string, any $N=4$ and $N=2$ orbifold sectors will lead to compactification moduli. In these models, the thresholds, $\Delta_{i}$, can be expressed roughly in terms of the moduli from the $N=2$ sectors as $[0,10,11]$

$$
\Delta_{i}(T, \bar{T}) \approx-b_{i} \log \left[(T+\bar{T})|\eta(T)|^{4}\right]
$$

where the complex modulus, $T$, is composed of the radius and an internal axion field of the 2 -torus fixed in an $N=2$ sector. This expression is pretty much fixed by the requirement of target space modular invariance. By absorbing this threshold into a redefinition of $M_{\mathrm{U}}$, we can interpret (4.1) as indicating that the effective coupling constants, $g_{i}$, only start running below a radius dependent scale, $M_{\mathrm{U}}$ [4,24].

On the other hand, for these type II models, all moduli are fixed to be at the point where the gauge symmetry is enlarged. As a result, there are no free parameters in the thresholds that can be adjusted to give large corrections. This feature of type II models would seem to indicate that the effective unification scale cannot easily be lowered much below (2.24). However, this should not be viewed as a drawback since the couplings are no longer required to meet at a point at the unification scale.

We have examined the running of the effective coupling constants and one-loop threshold effects in four-dimensional type II string theory as a possible first step towards relating strings to low-energy phenomenology. Our interest in studying the type II models is that it provides a simpler framework than the heterotic string where the fundamental ideas of unification can be understood. Work is in progress to develop more realistic type II models. When this is completed, it would be a simple matter to compute the gauge $\beta$-functions, $b_{i}$, from the massless particle spectrum and the thresholds, $\Delta_{i}$, from (2.22). This would then be sufficient for predicting the low-energy parameters $\sin ^{2} \theta_{W}$ and $\alpha_{s}$ at the $Z^{0}$ mass which may eventually lead to some testable results of string theory.

This work is supported in part by the U.S. Department of Energy under Grant No. DEFG05-85ER-40219. 


\section{References}

[1] J. Ellis, S. Kelley and D. V. Nanopoulos, Phys. Lett. B260 (1991) 131.

[2] U. Amaldi, W. de Boer and H. Fürstenau, Phys. Lett. B260 (1991) 447.

[3] P. Langacker and M. Luo, Phys. Rev. D44 (1991) 817.

[4] T. R. Taylor and G. Veneziano, Phys. Lett. B212 (1988) 147.

[5] J. A. Minahan, Nucl. Phys. B298 (1988) 36.

[6] V. S. Kaplunovsky, Nucl. Phys. B307 (1988) 145;

Errata, preprint hep-th@xxx/9205068 (May 1992).

[7] L. J. Dixon, V. S. Kaplunovsky and J. Louis, Nucl. Phys. B355 (1991) 649.

[8] L. E. Ibáñez, D. Lüst and G. G. Ross, Phys. Lett. B272 (1991) 251.

[9] I. Antoniadis, J. Ellis, R. Lacaze and D. V. Nanopoulos, Phys. Lett. B268 (1991) 188.

[10] A. Font, L. E. Ibáñez, D. Lüst and F. Quevedo, Phys. Lett. B245 (1990) 401.

[11] S. Ferrara, N. Magnoli, T. R. Taylor and G. Veneziano, Phys. Lett. B245 (1990) 409.

[12] I. Antoniadis, K. S. Narain and T. R. Taylor, Phys. Lett. B267 (1991) 37.

[13] J. A. Casas and C. Muñoz, Phys. Lett. B271 (1991) 85.

[14] L. J. Dixon, V. S. Kaplunovsky and C. Vafa, Nucl. Phys. B294 (1987) 43.

[15] P. Ginsparg, Phys. Lett. B197 (1987) 139.

[16] L. F. Abbott, Nucl. Phys. B185 (1981) 189.

[17] C. G. Callan, D. Friedan, E. J. Martinec and M. J. Perry, Nucl. Phys. B262 (1985) 593.

[18] R. Bluhm, L. Dolan and P. Goddard, Nucl. Phys. B289 (1987) 364.

[19] R. Bluhm, L. Dolan and P. Goddard, Nucl. Phys. B309 (1988) 330.

[20] I. Antoniadis, C. Bachas and C. Kounnas, Nucl. Phys. B289 (1987) 87.

[21] H. Kawai, D. Lewellen, J. A. Schwartz and H. Tye, Nucl. Phys. B299 (1988) 431.

[22] S. Weinberg, Phys. Lett. 91B (1980) 51.

[23] P. S. Howe, K. S. Stelle and P. C. West, Phys. Lett. 124B (1983) 55.

[24] I. Antoniadis, Phys. Lett. B246 (1990) 377. 